Sir,

\section{Corneal autografts: are the theoretical advantages achieved in practice?}

Corneal autografts offer potential advantages over conventional homografts in selected patients requiring penetrating keratoplasty, in particular the lack of the risk of immunological graft rejection. To be considered, one eye must have potentially useful vision limited by a diseased cornea and the fellow eye must have no useful vision but a healthy cornea. This unusual combination of clinical features means that corneal autografting is very rarely carried out, and remains virtually unreported. We report 4 cases where corneal autografts have restored useful vision.

\section{Case reports}

Case 1. A 70-year-old man had failure due to graft rejection of a conventional corneal homograft in the right eye. The graft had been carried out for bullous keratopathy following previous cataract extraction and lens implantation. He was under follow-up for advanced chronic simple glaucoma and had previously had bilateral trabeculectomies.

Despite potential limitation of final vision due to the advanced glaucoma, a further graft was considered appropriate for the right eye as his vision was only HM right eye, NPL left eye. In view of the previous graft failure and difficulties with compliance with postoperative topical treatment, it was decided to perform an autograft to the right eye using the patient's left cornea as donor.

A $9 \mathrm{~mm}$ donor button was harvested from the left eye using a Hessburg-Barron trephine and sutured to a $9 \mathrm{~mm}$ corneal trephine in the right eye with continuous 10-0 mersilene. A homograft was used for the left eye to avoid transposing the right failed corneal graft. There were no operative or post-operative complications. At 14 months follow-up the right cornea remains clear and the visual acuity with spectacle correction of $-4.00 /-2.75 \times 55$ is $6 / 60$.

Case 2. A 40-year-old man presented with vision of HM left eye and PL in the right eye. Eight years previously he had been injured by a grenade, with extensive facial and eye injuries. The left cornea was deeply scarred. The right cornea was intact but the eye was blind from traumatic optic neuropathy.

The patient underwent transposition of $7 \mathrm{~mm}$ corneal buttons using 10-0 nylon continuous suturing. There were no operative or post-operative complications. At 6 months follow-up the visual acuity in the left eye was $6 / 12$ with spectacles.
Case 3. A 51-year-old man developed a scarred, vascularised left cornea due to staphylococcal keratitis with vision of HM. The patient was a high myope with substantial chorioretinal degeneration, had glaucoma and type II diabetes and had a prior history of left herpetic kerato-uveitis. A psychiatric disorder limited compliance with treatment. The right eye was blind from previous eye trauma and end-stage glaucoma.

The patient underwent transposition of $7.5 \mathrm{~mm}$ corneal buttons sutured with continuous $10-0$ mersilene. Post-operatively the patient developed a small iris prolapse in the right eye which was managed conservatively. At his 3 month follow-up visit the left eye had vision of CF with a clear graft but the patient died shortly afterwards.

Case 4. A 63-year-old man developed right corneal decompensation and vision of only HM. He was a high myope and had type II diabetes. Both eyes were aphakic having had intracapsular cataract extraction aged 37 years. The left eye was blind from subsequent retinal detachment. On the right, proliferative retinopathy with vitreous haemorrhage had been treated over a 2 year period with vitrectomy, peripheral cryotherapy and repeat vitrectomy and endolaser. A Molteno tube had been inserted for uncontrolled intraocular pressure.

The patient underwent a right autograft using the healthy left cornea as donor. An $11 \mathrm{~mm}$ donor button was sutured with 10-0 interrupted nylon and 11-0 continuous mersilene sutures. In view of potential discomfort from the decompensated right cornea the left eye received a homograft. After 3 years follow-up the right cornea remains clear and the eye has vision of $6 / 60$ with spectacle correction of $+12.00 /-2.50 \times 120$ (Fig. 1).

\section{Comment}

The cases outlined demonstrate that useful vision can be successfully restored using corneal autografts. In each case there was an improvement in vision with the final

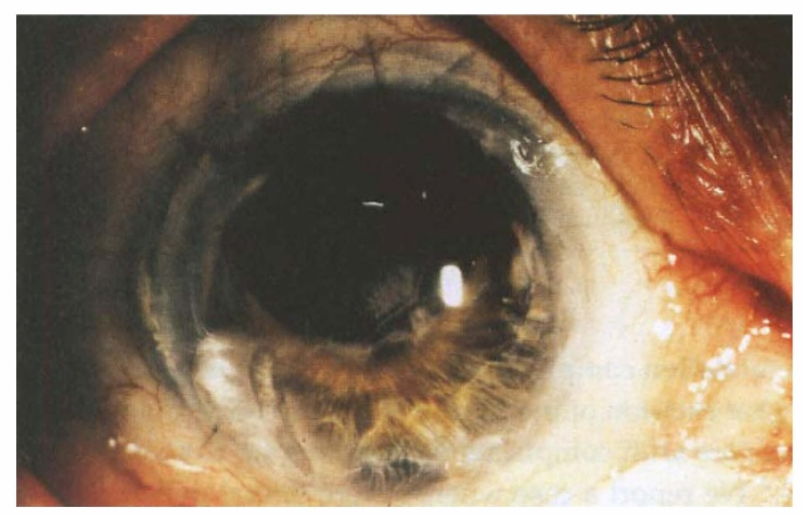

Fig. 1. Case 4. After 3 years follow-up the cornea remains clear and the eye has vision of $6 / 60$ with spectacle correction. 
level attained limited by coexistent posterior segment disease in 3 of the 4 patients.

Autografts avoid the risk of immunological graft rejection. Although this is an important advantage for any patient requiring a corneal graft, it may be of particular benefit where poor compliance with postoperative topical medication is likely or where access to ophthalmic services is limited. The need for prolonged and frequent follow-up is also likely to be reduced. In countries where donor corneas are unavailable corneal autografts may offer the only means of restoring vision to an eye with a diseased cornea if the fellow eye has no visual potential but a healthy cornea.

The unusual combination of clinical features that need to be present to consider autografting means that the procedure is rarely carried out. Hodkin and Insler ${ }^{11}$ reported a case in which a homograft had failed in an eye with previous stromal keratitis, the fellow eye being blind from traumatic optic neuropathy. The eye achieved a pinhole visual acuity of $6 / 12$. Tole $e t$ al. ${ }^{2}$ reported a case in which autografting of the cornea was combined with a limbal stem cell transplant in an eye that had sustained a severe alkali injury, the fellow eye being removed due to a large choroidal melanoma. The patient achieved a visual acuity of better than 6/9.

Oplinger et al..$^{3}$ reported 4 cases and warned of the risk of high post-operative astigmatism. However, 3 of the 4 cases had less than 7 dioptres of astigmatism. Significant post-operative astigmatism remains a problem in all corneal graft surgery and is unlikely to be exacerbated by using an autograft. Indeed, because of the lack of the risk of rejection, large-diameter autografts may be used safely and this may reduce the risk of significant post-operative astigmatism.

In summary, corneal autografting avoids the risk of graft rejection and in selected patients is successful in restoring useful vision.

\section{References}

1. Hodkin MJ, Insler MS. Transplantation of corneal tissue from a blind eye to a high-risk fellow eye by bilateral penetrating keratoplasty. Am J Ophthalmol 1994;117:808-9.

2. Tole DM, Edrich CL, Noble BA. Combined limbal and corneal autograft transplantation. Eye 1999;13:117-9.

3. Oplinger NL, Zaidman GW, Buxton DF. A comparison of corneal autografts with homografts. Ophthalmic Surg Lasers 1998;29:305-8.

\section{A.R. Pearson}

J.H. Sandford-Smith

Department of Ophthalmology

Leicester Royal Infirmary

Leicester LE1 5WW, UK

\section{Sir,}

Scleral dellen complicating primary pterygium excision Surgical excision of pterygium is a commonly performed procedure with complication rates ranging from $0^{1}$ to $26 \% .^{2}$ We report a case where severe scleral dellen resulted as a complication of bare sclera excision of a primary pterygium.
Case report

A 36-year-old plumber presented with a 6 month history of extreme irritation of both eyes. His working environment was dusty with frequent use of blow torches. Visual acuity was $6 / 5$ in both eyes. Eye examination was normal apart from bilateral pterygia. Notably his aqueous tear production was normal (Schirmer's test: $12 \mathrm{~mm}$ in $5 \mathrm{~min}$ ) as was the quality of his tear film.

Under local anaesthetic (lignocaine 2\% with adrenaline) the right nasal pterygium was excised in its entirety with subjacent tissue down to bare sclera (minimal cautery used). Following discharge the patient was prescribed oc. Betnesol-N (Evans) q.d.s. On review 2 weeks following surgery, the patient complained of a 2 day history of severe pain in the right eye. Slit-lamp examination revealed a large, deep dellen centred on the bare sclera surrounded by oedematous conjunctiva. The ciliary body was visible through the thin sclera. Management involved covering the dellen with a conjunctival flap and regular antibiotic ointment.

Apart from the drying effect produced by the oedematous conjunctiva, no other aetiological factor was found. History, clinical examination and laboratory tests (including full blood count, ESR, serum rheumatoid factor and autoantibody screening) failed to reveal autoimmune inflammatory disease or gout.

\section{Comment}

Surgical management of pterygia involves bare sclera excision with or without adjunctive therapy. Recurrence rates after bare sclera excision alone vary from $32 \%{ }^{2}$ to $88 \%,{ }^{3}$ suggesting that a number of factors play a role in producing these disparate results. Race, patient age, latitude, ${ }^{3,4}$ experience of the surgeon, severe tear film abnormality and return to unfavourable working conditions, ${ }^{4}$ are all suggested explanatory factors for the variation in reported recurrence rates.

Adjunctive treatments include: beta irradiation, conjunctival autograft and mitomycin-C. Beta irradiation has mostly fallen out of favour because of its severe complications. ${ }^{5}$ Post-operative mitomycin-C drops have,

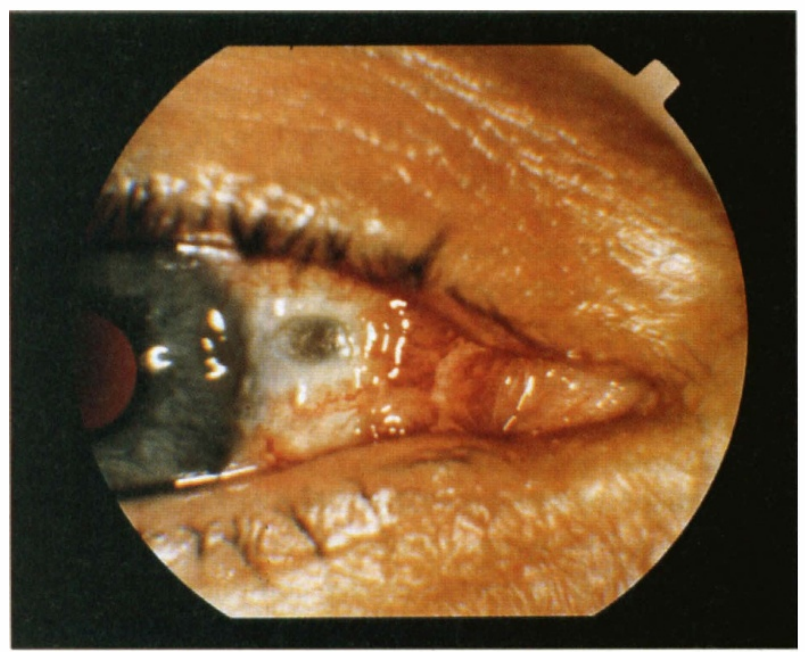

Fig. 1. Severe scleral dellen as a complication of bare sclera excision. 